\title{
Pata-Type Fixed-Point Theorems in Kaleva-Seikkala's Type Fuzzy Metric Space
}

\author{
Ao-Lei Sima, Fei He $\mathbb{B}$, and Ning Lu \\ School of Mathematical Sciences, Inner Mongolia University, Hohhot 010021, China \\ Correspondence should be addressed to Fei He; hefei@imu.edu.cn
}

Received 26 September 2019; Revised 7 January 2020; Accepted 29 January 2020; Published 28 February 2020

Academic Editor: Giuseppe Marino

Copyright ( 2020 Ao-Lei Sima et al. This is an open access article distributed under the Creative Commons Attribution License, which permits unrestricted use, distribution, and reproduction in any medium, provided the original work is properly cited.

The purpose of this paper is to generalize the fixed-point theorems for Banach-Pata-type contraction and Kannan-Pata-type contraction from metric spaces to Kaleva-Seikkala's type fuzzy metric spaces. Moreover, two examples are given for the support of our results.

\section{Introduction and Preliminaries}

In 1984, Kaleva and Seikkala [1] introduced the concept of fuzzy metric spaces for the first time, and they established some fixed-point theorems in a complete fuzzy metric space. This result was investigated by many authors from different points of view, see [2-10] and the references therein.

Definition 1 (see [11]). A mapping $\eta: \mathbb{R} \longrightarrow[0,1]$ is called a fuzzy real number, whose $\alpha$ - level set is denoted by $[\eta]_{\alpha}=$ $\{t \in \mathbb{R}: \eta(t) \geq \alpha\}$ if it satisfies two axioms:

(1) There exists $t_{0} \in \mathbb{R}$ such that $\eta\left(t_{0}\right)=1$

(2) For each $\alpha \in(0,1],[\eta]_{\alpha}=\left[\lambda_{\alpha}, \rho_{\alpha}\right]$ is a closed interval of $\mathbb{R}$, where $-\infty<\lambda_{\alpha} \leq \rho_{\alpha}<+\infty$

The set of all such fuzzy real numbers is denoted by $\mathscr{F}$. If $\eta \in \mathscr{F}$ and $\eta(q)=0$ whenever $q<0$, then $\eta$ is called a nonnegative fuzzy real number, and by $\mathscr{F}^{+}$, we mean the set of all nonnegative fuzzy real numbers. The notation $\overline{0}$ stands for the fuzzy number satisfying $\overline{0}(t)=1$ and $\overline{0}(t)=0$ if $t \neq 0$. Clearly, $\overline{0} \in \mathscr{F}^{+}$. $\mathbb{R}$ can be embedded in $\mathscr{F}$ if $a \in \mathscr{F}$ satisfies $\bar{a}(q)=\overline{0}(q-a)$.

Definition 2 (see [1]). Let $X$ be a nonempty set and the mappings $L, R:[0,1] \times[0,1] \longrightarrow[0,1]$ be symmetric, nondecreasing in both arguments, and satisfy $L(0,0)=0$, $R(1,1)=1$. Let $d$ be the mapping $X \times X \longrightarrow \mathscr{F}^{+}$, and write $[d(x, y)]_{\alpha}=\left[\lambda_{\alpha}(x, y), \rho_{\alpha}(x, y)\right]$ for all $x, y \in X$ and all $\alpha \in(0,1]$. The quadruple $(X, d, L, R)$ is called a fuzzy metric space if the following axioms are satisfied:

(D1) $d(x, y)=\overline{0} \Longleftrightarrow x=y$.

(D2) for all $x, y \in X, d(x, y)=d(y, x)$.

(D3) for all $x, y, z \in X$.

(D3L) whenever $p \leq \lambda_{1}(x, z), q \leq \lambda_{1}(z, y)$, and $p+q \leq$ $\lambda_{1}(x, y), d(x, y)(p+q) \geq L(d(x, z)(p), d(z, y)(q))$.

(D3R) whenever $p \geq \lambda_{1}(x, z), q \geq \lambda_{1}(z, y)$, and $p+q \geq$ $\lambda_{1}(x, y), d(x, y)(p+q) \leq R(d(x, z)(p), d(z, y)(q))$.

Lemma 1 (see [6]). Let $(X, d, L, R)$ be a fuzzy metric space and $[d(x, y)]_{t}=\left[\lambda_{t}(x, y), \rho_{t}(x, y)\right]$ for all $t \in(0,1]$, where $x, y \in X$ are any two fixed elements. Then,

(1) $\lim _{q \longrightarrow-\infty} d(x, y)(q)=\lim _{q \longrightarrow+\infty} d(x, y)(q)=0$

(2) $d(x, y)(q)$ is a left continuous and nonincreasing function for $q \in\left(\lambda_{1}(x, y),+\infty\right)$

(3) $\rho_{t}(x, y)$ is a left continuous and nonincreasing function for $t \in(0,1]$

Lemma 2 (see [12]). Let $(X, d, L, R)$ be a fuzzy metric space, and suppose that

$$
(R-1) R(a, b) \leq \max \{a, b\}
$$


$(R-2)$ for each $t \in(0,1]$, there exists $s \in(0, t]$ such that $R(s, r)<t$ for all $r \in(0, t)$

$(R-3) \lim _{t \rightarrow 0^{+}} R(t, t)=0$

Then, $(R-1) \Rightarrow(R-2) \Rightarrow(R-3)$.

Lemma 3 (see [6]). Let $(X, d, L, R)$ be a fuzzy metric space. Then,

(1) (R-1) implies that, for each $t \in(0,1]$,

$$
\rho_{t}(x, y) \leq \rho_{t}(x, z)+\rho_{t}(z, y)
$$

for all $x, y, z \in X$.

(2) $(R-2)$ implies that, for each $t \in(0,1]$, there exists $s=$ $s(t) \in(0, t]$ such that

$$
\rho_{t}(x, y) \leq \rho_{s}(x, z)+\rho_{t}(z, y)
$$

for all $x, y, z \in X$.

(3) (R-3) implies that, for each $t \in(0,1]$, there exists $s=$ $s(t) \in(0, t]$ such that

$$
\rho_{t}(x, y) \leq \rho_{s}(x, z)+\rho_{s}(z, y)
$$

for all $x, y, z \in X$.

Definition 3 (see [6]). Let $(X, d, L, R)$ be a fuzzy metric space and $\left\{x_{n}\right\} \subset X, x \in X$. Then,

(1) $\left\{x_{n}\right\}$ is said to be convergent to $x$ if $\lim _{n \longrightarrow \infty} d\left(x_{n}, x\right)=\overline{0}$, i.e., $\lim _{n \longrightarrow \infty} \rho_{t}\left(x_{n}, x\right)=0$ for all $t \in(0,1]$

(2) $\left\{x_{n}\right\}$ is called a Cauchy sequence in $X$ if $\lim _{n, m \rightarrow \infty} d\left(x_{n}, x_{m}\right)=\overline{0}$; equivalently, for any given $\varepsilon>0$ and $t \in(0,1]$, there exists $N=N(\varepsilon, t) \in \mathbb{N}$ such that $\rho_{t}\left(x_{n}, x_{m}\right)<\varepsilon$ whenever $n, m \geq N$

(3) $(X, d, L, R)$ is said to be complete if each Cauchy sequence in $X$ is convergent to some point in $X$

Lemma 4 (see [6]). Let $(X, d, L, R)$ be a fuzzy metric space with $(R-2)$. Then, for each $t \in(0,1], \rho_{t}(x, y)$ is continuous at $(x, y) \in X \times X$.

In 2011, Pata [13] extended the Banach contraction principle with weaker hypotheses than those of the Banach contraction principle in the complete metric space. Since then, several other fixed point results in the spirit of Pata have appeared, see [14-18]. In particular, Chakraborty and Samanta [14] proved a generalization of Kannan's fixedpoint theorem based on the result of Pata.

Throughout the following, $(X, d)$ will be a complete metric space, and $(X, d, L, R)$ will be a complete fuzzy metric space. Fix an arbitrary point $x_{0} \in X$, and we denote $\|x\|=$ $d\left(x, x_{0}\right)$ and $\|x\|_{t}=\rho_{t}\left(x, x_{0}\right)$ for all $x \in X$ and $t \in(0,1]$. Also, $\psi:[0,1] \longrightarrow[0, \infty)$ is an increasing function, continuous at zero, with $\psi(0)=0$. Given a function $f: X \longrightarrow X$.
Theorem 1 (see [13]). Let $(X, d)$ be a complete metric space. Let $\Lambda \geq 0, \alpha \geq 1$, and $\beta \in[0, \alpha]$ be fixed constants. If the inequality

$$
d(f x, f y) \leq(1-\varepsilon) d(x, y)+\Lambda \varepsilon^{\alpha} \psi(\varepsilon)[1+\|x\|+\|y\|]^{\beta},
$$

is satisfied for every $\varepsilon \in[0,1]$ and all $x, y \in X$, then $f$ has $a$ unique fixed point $z \in X$.

Theorem 2 (see [14]). Let $(X, d)$ be a complete metric space. Let $\Lambda \geq 0, \alpha \geq 1$, and $\beta \in[0, \alpha]$ be fixed constants. If the inequality

$$
\begin{aligned}
d(f x, f y) \leq & \frac{1-\varepsilon}{2}[d(x, f x)+d(y, f y)]+\Lambda \varepsilon^{\alpha} \psi(\varepsilon)[1+\|x\| \\
& +\|y\|+\|f x\|+f y]^{\beta},
\end{aligned}
$$

is satisfied for every $\varepsilon \in[0,1]$ and all $x, y \in X$, then $f$ has a unique fixed point $z \in X$.

In this paper, we prove two further extensions of Patatype fixed theorems in complete Kaleva-Seikkala's type fuzzy metric space using contractive condition of Banach type and Kannan type. Afterwards, the fixed-point theorems for the corresponding linear contraction are given as corollaries. Our theorems extend the main results of $[13,14]$. Moreover, two nontrivial examples are given to illustrate our two theorems, and our examples show that these two theorems are independent to each other.

\section{Main Results}

Our results of this paper are stated as follows.

Theorem 3. Let $(X, d, L, R)$ be a complete fuzzy metric space with (R-2). Let $\Lambda \geq 0$ and $\alpha \geq 1$ be fixed constants. Let $f: X \longrightarrow X$ be a mapping such that

$$
\begin{aligned}
\rho_{t}(f x, f y) \leq & (1-\varepsilon) \rho_{t}(x, y)+\Lambda \varepsilon^{\alpha} \psi(\varepsilon)\left[1+\|x\|_{t}+\|y\|_{t}\right. \\
& \left.+\|f x\|_{t}+\|f y\|_{t}\right]^{\alpha},
\end{aligned}
$$

for every $\varepsilon \in[0,1], t \in(0,1]$ and all $x, y \in X$. Then, $f$ has $a$ unique fixed point $z \in X$.

Proof. Starting from $x_{0}$, construct a sequence $\left\{x_{n}\right\}$ such that $x_{n}=f x_{n-1}=f^{n} x_{0}$. For all $t \in(0,1]$, we denote $C_{n}(t)=$ $\left\|x_{n}\right\|_{t}$. If $x_{n_{0}}=x_{n_{0}+1}$ for some $n_{0}$, then $x_{n_{0}}$ is a fixed point of $f$. Thus, we always assume that $x_{n} \neq x_{n+1}$ for all $n \in \mathbb{N}$.

In order to prove this theorem, we divide into the following five steps:

Step 1. We show that the sequence $\left\{\rho_{t}\left(x_{n+1}, x_{n}\right)\right\}$ is decreasing. Clearly, suppose that $t \in(0,1]$ and $\varepsilon=0$ in (6), we obtain 


$$
\rho_{t}\left(x_{n+1}, x_{n}\right) \leq \rho_{t}\left(x_{n}, x_{n-1}\right) \leq \cdots \leq \rho_{t}\left(x_{1}, x_{0}\right) .
$$

Step 2. We prove that the sequence $\left\{C_{n}(t)\right\}$ is bounded. For any $t \in(0,1]$, by Lemma 3 , there exists $s=s(t) \in(0, t]$ such that

$$
\rho_{t}(x, y) \leq \rho_{s}(x, z)+\rho_{t}(z, y),
$$

for all $x, y, z \in X$. Then, from Step 1 , we have

$$
\begin{aligned}
C_{n}(t)= & \rho_{t}\left(x_{n}, x_{0}\right) \\
\leq & \rho_{s}\left(x_{n}, x_{n+1}\right)+\rho_{s}\left(x_{1}, x_{0}\right)+\rho_{t}\left(x_{n+1}, x_{1}\right) \\
\leq & \rho_{t}\left(x_{n+1}, x_{1}\right)+2 \rho_{s}\left(x_{1}, x_{0}\right) \\
\leq & (1-\varepsilon) \rho_{t}\left(x_{n}, x_{0}\right)+\Lambda \varepsilon^{\alpha} \psi(\varepsilon)\left[1+\left\|x_{n}\right\|_{t}+\left\|x_{0}\right\|_{t}\right. \\
& \left.+\left\|x_{n+1}\right\|_{t}+\left\|x_{1}\right\|_{t}\right]^{\alpha}+2 \rho_{s}\left(x_{1}, x_{0}\right) \\
= & (1-\varepsilon) \rho_{t}\left(x_{n}, x_{0}\right)+\Lambda \varepsilon^{\alpha} \psi(\varepsilon)\left[1+C_{n}(t)+C_{n+1}(t)\right. \\
& \left.+C_{1}(t)\right]^{\alpha}+2 \rho_{s}\left(x_{1}, x_{0}\right) .
\end{aligned}
$$

Since $\quad C_{n+1}(t) \leq \rho_{s}\left(x_{n+1}, x_{n}\right)+\rho_{t}\left(x_{n}, x_{0}\right) \leq \rho_{s}\left(x_{1}, x_{0}\right)+$ $\rho_{t}\left(x_{n}, x_{0}\right)$, we have

$$
\begin{aligned}
C_{n}(t) \leq & (1-\varepsilon) C_{n}(t)+\Lambda \varepsilon^{\alpha} \psi(\varepsilon)\left[1+2 C_{n}(t)+C_{1}(t)\right. \\
& \left.+\rho_{s}\left(x_{1}, x_{0}\right)\right]^{\alpha}+2 \rho_{s}\left(x_{1}, x_{0}\right) \\
\leq & (1-\varepsilon) C_{n}(t)+\Lambda \varepsilon^{\alpha} \psi(\varepsilon)\left[1+2 C_{n}(t)+2 \rho_{s}\left(x_{1}, x_{0}\right)\right]^{\alpha} \\
& +2 \rho_{s}\left(x_{1}, x_{0}\right) .
\end{aligned}
$$

Hence,

$$
\varepsilon C_{n}(t)-2 \rho_{s}\left(x_{1}, x_{0}\right) \leq \Lambda \varepsilon^{\alpha} \psi(\varepsilon)\left[1+2 C_{n}(t)+2 \rho_{s}\left(x_{1}, x_{0}\right)\right]^{\alpha} .
$$

Suppose that $\left\{C_{n}(t)\right\}$ is unbounded. Then, there exist $t_{0} \in(0,1], s_{0} \in\left(0, t_{0}\right]$, and a subsequence $\left\{C_{n_{k}}\left(t_{0}\right)\right\}$ of $\left\{C_{n}\left(t_{0}\right)\right\}$ such that $C_{n_{k}}\left(t_{0}\right) \longrightarrow \infty(k \longrightarrow \infty)$ and $C_{n_{k}}\left(t_{0}\right) \geq 1+2 \rho_{s_{0}}\left(x_{0}, x_{1}\right)$. Let

$$
\varepsilon=\varepsilon_{k}=\frac{1+2 \rho_{s_{0}}\left(x_{1}, x_{0}\right)}{C_{n_{k}}\left(t_{0}\right)} .
$$

Then, we get

$$
\begin{aligned}
& \frac{1+2 \rho_{s_{0}}\left(x_{1}, x_{0}\right)}{C_{n_{k}}\left(t_{0}\right)} C_{n_{k}}\left(t_{0}\right)-2 \rho_{s_{0}}\left(x_{1}, x_{0}\right) \\
& \leq \Lambda \varepsilon_{k}^{\alpha} \psi\left(\varepsilon_{k}\right)\left[1+2 C_{n_{k}}\left(t_{0}\right)+2 \rho_{s_{0}}\left(x_{1}, x_{0}\right)\right]^{\alpha} \\
& =\Lambda\left[\left(1+2 \rho_{s_{0}}\left(x_{1}, x_{0}\right)\right) \frac{1+2 C_{n_{k}}\left(t_{0}\right)+2 \rho_{s_{0}}\left(x_{1}, x_{0}\right)}{C_{n_{k}}\left(t_{0}\right)}\right]^{\alpha} \psi\left(\varepsilon_{k}\right),
\end{aligned}
$$

which implies that

$$
1 \leq \Lambda\left[\left(1+2 \rho_{s_{0}}\left(x_{1}, x_{0}\right)\right) \frac{1+2 C_{n_{k}}\left(t_{0}\right)+2 \rho_{s_{0}}\left(x_{1}, x_{0}\right)}{C_{n_{k}}\left(t_{0}\right)}\right]^{\alpha} \psi\left(\varepsilon_{k}\right) .
$$

Letting $k \longrightarrow \infty$ in (14), we have $\psi\left(\varepsilon_{k}\right) \longrightarrow 0$ and

$$
\Lambda\left(1+2 \rho_{s_{0}}\left(x_{1}, x_{0}\right)\right)^{\alpha}\left[\frac{1+C_{1}\left(t_{0}\right)+2 \rho_{s_{0}}\left(x_{1}, x_{0}\right)}{C_{n_{k}}\left(t_{0}\right)}+2\right]^{\alpha}
$$

$$
\psi\left(\varepsilon_{k}\right) \longrightarrow 0
$$

which contradict (14). Thus, $\left\{C_{n}(t)\right\}$ is bounded, that is, for any $t \in(0,1]$, there exists a constant $M_{t}>0$ such that $\left\|x_{n}\right\|_{t}=C_{n}(t) \leq M_{t}$.

Step 3. We shall show that

$$
\lim _{n \longrightarrow \infty} \rho_{t}\left(x_{n+1}, x_{n}\right)=0(t \in(0,1]) .
$$

Note that $\left\{\rho_{t}\left(x_{n+1}, x_{n}\right)\right\}$ is a decreasing and bounded sequence. So, assume that $\lim _{n \longrightarrow \infty} \rho_{t_{0}}\left(x_{n+1}, x_{n}\right)=a$ for some $a>0$ and $t_{0} \in(0,1]$. By (6), we obtain that

$$
\begin{aligned}
\rho_{t_{0}}\left(x_{n+1}, x_{n}\right)= & \rho_{t_{0}}\left(f x_{n}, f x_{n-1}\right) \\
\leq & (1-\varepsilon) \rho_{t_{0}}\left(x_{n}, x_{n-1}\right)+\Lambda \varepsilon^{\alpha} \psi(\varepsilon)\left[1+\left\|x_{n}\right\|_{t_{0}}\right. \\
& \left.+\left\|x_{n-1}\right\|_{t_{0}}+\left\|x_{n+1}\right\|_{t_{0}}+\left\|x_{n}\right\|_{t_{0}}\right]^{\alpha} \\
\leq & (1-\varepsilon) \rho_{t_{0}}\left(x_{n}, x_{n-1}\right)+\Lambda \varepsilon^{\alpha} \psi(\varepsilon)\left[1+4 M_{t_{0}}\right]^{\alpha} .
\end{aligned}
$$

Letting $n \longrightarrow \infty$, we have

$$
a \leq \Lambda \varepsilon^{\alpha-1} \psi(\varepsilon)\left[1+4 M_{t_{0}}\right]^{\alpha} .
$$

Note that

$$
\Lambda \varepsilon^{\alpha-1} \psi(\varepsilon)\left[1+4 M_{t_{0}}\right]^{\alpha} \longrightarrow 0 \text { as } \varepsilon \longrightarrow 0 .
$$

Then, we can see $a \leq 0$, which contradicts our assumption.

Step 4. We show that $\left\{x_{n}\right\}$ is a Cauchy sequence. Suppose not, choose $\delta>0$ and $t_{0} \in(0,1]$, and then there exist subsequences $\left\{x_{n_{k}}\right\}$ and $\left\{x_{m_{k}}\right\}$ of $\left\{x_{n}\right\}$ with $k<m_{k}<n_{k}$ such that

$$
\rho_{t_{0}}\left(x_{m_{k}}, x_{n_{k}}\right)>\delta \text { and } \rho_{t_{0}}\left(x_{m_{k}}, x_{n_{k}-1}\right) \leq \delta \text {. }
$$

By Lemma 3, there exists $s_{0} \in\left(0, t_{0}\right]$ such that

$$
\begin{aligned}
\delta< & \rho_{t_{0}}\left(x_{m_{k}}, x_{n_{k}}\right) \leq \rho_{t_{0}}\left(x_{m_{k}}, x_{n_{k}-1}\right)+\rho_{s_{0}}\left(x_{n_{k}-1}, x_{n_{k}}\right) \\
& \leq \rho_{s_{0}}\left(x_{n_{k}-1}, x_{n_{k}}\right)+\delta .
\end{aligned}
$$

Putting $n \longrightarrow \infty$, we get $\rho_{t_{0}}\left(x_{m_{k}}, x_{n_{k}}\right) \longrightarrow \delta$. Similarly, we can see that 


$$
\begin{aligned}
\rho_{t_{0}}\left(x_{n_{k}+1}, x_{m_{k}+1}\right) \leq & \rho_{t_{0}}\left(x_{n_{k}}, x_{m_{k}}\right)+\rho_{s_{0}}\left(x_{n_{k}}, x_{n_{k}+1}\right) \\
& +\rho_{s_{0}}\left(x_{m_{k}}, x_{m_{k}+1}\right), \\
\rho_{t_{0}}\left(x_{n_{k}+1}, x_{m_{k}+1}\right) \geq & \rho_{t_{0}}\left(x_{n_{k}}, x_{m_{k}}\right)-\rho_{s_{0}}\left(x_{n_{k}}, x_{n_{k}+1}\right) \\
& -\rho_{s_{0}}\left(x_{m_{k}}, x_{m_{k}+1}\right) .
\end{aligned}
$$

Passing to the limit as $n \longrightarrow \infty$ in (22) and (23), we have $\rho_{t_{0}}\left(x_{n_{k}+1}, x_{m_{k}+1}\right) \longrightarrow \delta$. By (6), we get

$$
\begin{aligned}
\rho_{t_{0}}\left(x_{n_{k}+1}, x_{m_{k}+1}\right)= & \rho_{t_{0}}\left(f x_{n_{k}}, f x_{m_{k}}\right) \\
\leq & (1-\varepsilon) \rho_{t_{0}}\left(x_{n_{k}}, x_{m_{k}}\right)+\Lambda \varepsilon^{\alpha} \psi(\varepsilon)\left[1+\left\|x_{n_{k}}\right\|_{t_{0}}\right. \\
& \left.\quad+\left\|x_{m_{k}}\right\|_{t_{0}}+\left\|x_{n_{k}+1}\right\|_{t_{0}}+\left\|x_{m_{k}+1}\right\|_{t_{0}}\right]^{\alpha} \\
\leq & (1-\varepsilon) \rho_{t_{0}}\left(x_{n_{k}}, x_{m_{k}}\right)+\Lambda \varepsilon^{\alpha} \psi(\varepsilon)\left[1+4 M_{t_{0}}\right]^{\alpha} .
\end{aligned}
$$

Taking $n \longrightarrow \infty$ in (2), we obtain that

$$
\begin{aligned}
& \delta \leq(1-\varepsilon) \delta+\Lambda \varepsilon^{\alpha} \psi(\varepsilon)\left[1+4 M_{t_{0}}\right]^{\alpha}, \\
& \delta \leq \Lambda \varepsilon^{\alpha-1} \psi(\varepsilon)\left[1+4 M_{t_{0}}\right]^{\alpha} .
\end{aligned}
$$

Note that $\Lambda \varepsilon^{\alpha-1} \psi(\varepsilon)\left[1+4 M_{t_{0}}\right]^{\alpha} \longrightarrow 0(\varepsilon \longrightarrow 0)$, a contradiction. Thus, $\left\{x_{n}\right\}$ is a Cauchy sequence. Since $X$ is complete, there exists $z \in X$ such that $x_{n} \longrightarrow z(n \longrightarrow \infty)$.

Step 5. We prove that $z$ is the unique fixed point for mapping $f$.

First, we show that $f z=z$. By $(6)$, for all $t \in(0,1]$, there exists $s \in(0, t]$, and we have

$$
\begin{aligned}
\rho_{t}(f z, z) \leq & \rho_{s}\left(x_{n+1}, z\right)+\rho_{t}\left(f z, x_{n+1}\right) \\
\leq & (1-\varepsilon) \rho_{t}\left(z, x_{n}\right)+\Lambda \varepsilon^{\alpha} \psi(\varepsilon)\left(1+\|z\|_{t}+\left\|x_{n}\right\|_{t}\right. \\
& \left.\quad+\|f z\|_{t}+\left\|x_{n+1}\right\|_{t}\right)^{\alpha}+\rho_{s}\left(x_{n+1}, z\right) \\
\leq & (1-\varepsilon) \rho_{t}\left(z, x_{n}\right)+\Lambda \varepsilon^{\alpha} \psi(\varepsilon)\left(1+4 M_{t}\right)^{\alpha}+\rho_{s}\left(x_{n+1}, z\right) .
\end{aligned}
$$

Letting $n \longrightarrow \infty$, we get $\rho_{t}(f z, z)=0$. Hence, $f z=z$.

Next, we prove the uniqueness of $z$. Assume that $z^{\prime}$ is another fixed point for $f$. For each $t \in(0,1]$, there exists $s \in(0, t]$ such that

$$
\begin{aligned}
\rho_{t}\left(z, z^{\prime}\right)= & \rho_{t}\left(f z, f z^{\prime}\right) \\
\leq & (1-\varepsilon) \rho_{t}\left(z, z^{\prime}\right)+\Lambda \epsilon^{\alpha} \psi(\varepsilon)\left[1+\|z\|_{t}+\left\|z^{\prime}\right\|_{t}\right. \\
& \left.+\|f\| z_{t}+\left\|f z^{\prime}\right\|_{t}\right]^{\alpha} \\
\leq & (1-\varepsilon) \rho_{t}\left(z, z^{\prime}\right)+\Lambda \epsilon^{\alpha} \psi(\varepsilon)\left[1+4 M_{t}\right]^{\alpha} .
\end{aligned}
$$

Thus, we have $\rho_{t}\left(z, z^{\prime}\right) \leq \Lambda \varepsilon^{\alpha-1} \psi(\varepsilon)\left[1+4 M_{t}\right]^{\alpha} \longrightarrow 0$ as $\varepsilon \longrightarrow 0$, which implies $z=z^{\prime}$. Therefore, $f$ has a unique fixed point $z \in X$.

Remark 1. From the proof of Theorem 3, we can see that, to keep the sequence $\left\{x_{n}\right\}$ converge to the fixed point, the range of $\varepsilon$ in (6) can be limited from $[0,1]$ to $[0, \gamma]$ for some given constant $\gamma \in(0,1]$.

By Remark 1, letting $\gamma=\lambda$, we can deduce the following corollary, which is the Banach contraction principle in fuzzy metric spaces.

Corollary 1 (see Corollary 5.3, [6]). Let $(X, d, L, R)$ be a complete fuzzy metric space with $(R-2)$ and $f: X \longrightarrow X$ be a mapping. If there exists $\lambda \in[0,1)$ such that

$$
\rho_{t}(f x, f y) \leq \lambda \rho_{t}(x, y)
$$

for all $t \in(0,1]$ and $x, y \in X$, then $f$ has a unique fixed point $z \in X$.

Theorem 4 generalizes the result in [14] to fuzzy metric spaces.

Theorem 4. Let $(X, d, L, R)$ be a complete fuzzy metric space with (R-2). Let $\Lambda \geq 0$ and $\alpha \geq 1$ be fixed constants. Let $f: X \longrightarrow X$ be a mapping such that

$$
\begin{aligned}
\rho_{t}(f x, f y) \leq & \frac{1-\varepsilon}{2}\left[\rho_{t}(x, f x)+\rho_{t}(y, f y)\right]+\Lambda \varepsilon^{\alpha} \psi(\varepsilon)[1 \\
& \left.+\|x\|_{t}+\|y\|_{t}+\|f x\|_{t}+\|f y\|_{t}\right]^{\alpha}
\end{aligned}
$$

for every $\varepsilon \in[0,1], t \in(0,1]$, and all $x, y \in X$. Then, $f$ has $a$ unique fixed point $z \in X$.

Proof. Starting from $x_{0}$, construct a sequence $\left\{x_{n}\right\}$ such that $x_{n}=f x_{n-1}=f^{n} x_{0}$. For any $t \in(0,1]$, we denote $C_{n}(t)=$ $\left\|x_{n}\right\|_{t}$. If $x_{n_{0}}=x_{n_{0}+1}$ for some $n_{0}$, then $x_{n_{0}}$ is a fixed point of $f$. Thus, we always assume that $x_{n} \neq x_{n+1}$ for all $n \in \mathbb{N}$. Then, the proof is divided into the following five steps:

Step 1. We show that the sequence $\left\{\rho_{t}\left(x_{n}, x_{n+1}\right)\right\}$ is decreasing. Suppose that $t \in(0,1]$ and $\varepsilon=0$ in (29), we obtain

$$
\rho_{t}\left(x_{n}, x_{n+1}\right) \leq \frac{1}{2}\left(\rho_{t}\left(x_{n-1}, x_{n}\right)+\rho_{t}\left(x_{n}, x_{n+1}\right)\right) \text {. }
$$

Thus, we get

$$
\rho_{t}\left(x_{n+1}, x_{n}\right) \leq \rho_{t}\left(x_{n}, x_{n-1}\right) \leq \cdots \leq \rho_{t}\left(x_{1}, x_{0}\right) .
$$

Step 2. The sequence $\left\{C_{n}(t)\right\}$ is bounded. For each $t \in(0,1]$, there exists $s=s(t) \in(0, t]$, and 


$$
\begin{aligned}
C_{n}(t)= & \rho_{t}\left(x_{n}, x_{0}\right) \\
\leq & \rho_{s}\left(x_{n}, x_{n+1}\right)+\rho_{s}\left(x_{1}, x_{0}\right)+\rho_{t}\left(x_{n+1}, x_{1}\right) \\
\leq & \rho_{t}\left(x_{n+1}, x_{1}\right)+2 \rho_{s}\left(x_{1}, x_{0}\right) \\
\leq & (1-\varepsilon) \frac{1}{2}\left(\rho_{t}\left(x_{n}, x_{n+1}\right)+\rho_{t}\left(x_{1}, x_{0}\right)\right) \\
& +\Lambda \varepsilon^{\alpha} \psi(\varepsilon)\left[1+\left\|x_{n}\right\|_{t}+\left\|x_{0}\right\|_{t}+\left\|x_{n+1}\right\|_{t}+\left\|x_{1}\right\|_{t}\right]^{\alpha} \\
& +2 \rho_{s}\left(x_{1}, x_{0}\right) \\
\leq & (1-\varepsilon) \rho_{t}\left(x_{1}, x_{0}\right)+\Lambda \varepsilon^{\alpha} \psi(\varepsilon)\left[1+C_{n}(t)\right. \\
& \left.+C_{n+1}(t)+C_{1}(t)\right]^{\alpha}+2 \rho_{s}\left(x_{1}, x_{0}\right) .
\end{aligned}
$$

Letting $\varepsilon=0$ in (32), there exists a constant $M_{t}>0$ such that $C_{n}(t) \leq \rho_{t}\left(x_{0}, x_{1}\right)=M_{t}$.

Step 3. We shall prove that $\lim _{n \longrightarrow \infty} \rho_{t}\left(x_{n}, x_{n+1}\right)=0$ for each $t \in(0,1]$. For any $t \in(0,1]$, we obtain

$$
\begin{aligned}
\rho_{t}\left(x_{n}, x_{n+1}\right)= & \rho_{t}\left(f x_{n-1}, f x_{n}\right) \\
\leq & (1-\varepsilon) \frac{1}{2}\left(\rho_{t}\left(x_{n-1}, x_{n}\right)+\rho_{t}\left(x_{n}, x_{n+1}\right)\right) \\
& +\Lambda \varepsilon^{\alpha} \psi(\varepsilon)\left[1+\left\|x_{n-1}\right\|_{t}+\left\|x_{n}\right\|_{t}+\left\|x_{n}\right\|_{t}+\left\|x_{n+1}\right\|_{t}\right]^{\alpha} \\
\leq & (1-\varepsilon) \frac{1}{2}\left(\rho_{t}\left(x_{n-1}, x_{n}\right)+\rho_{t}\left(x_{n}, x_{n+1}\right)\right) \\
& +\Lambda \varepsilon^{\alpha} \psi(\varepsilon)\left[1+4 M_{t}\right]^{\alpha} .
\end{aligned}
$$

Since $\left\{\rho_{t}\left(x_{n}, x_{n+1}\right)\right\}$ is a decreasing and bounded sequence, assume that $\lim _{n \longrightarrow \infty} \rho_{t}\left(x_{n}, x_{n+1}\right)=a$ for some $a>0$. Letting $n \longrightarrow \infty$ in (2), we have

$$
\begin{aligned}
a & \leq(1-\varepsilon) \frac{1}{2} \cdot 2 a+\Lambda \varepsilon^{\alpha} \psi(\varepsilon)\left[1+4 M_{t}\right]^{\alpha} \\
& \leq(1-\varepsilon) a+\Lambda \varepsilon^{\alpha} \psi(\varepsilon)\left[1+4 M_{t}\right]^{\alpha} \\
a & \leq \Lambda \varepsilon^{\alpha-1} \psi(\varepsilon)\left[1+4 M_{t}\right]^{\alpha} .
\end{aligned}
$$

From

$$
\Lambda \varepsilon^{\alpha-1} \psi(\varepsilon)\left[1+4 M_{t}\right]^{\alpha} \longrightarrow 0(\varepsilon \longrightarrow 0),
$$

we see $a \leq 0$, a contradiction. For each $t \in(0,1]$, we get $\rho_{t}\left(x_{n}, x_{n+1}\right) \longrightarrow 0(n \longrightarrow \infty)$.

Step 4. We show that $\lim _{n \longrightarrow \infty} \rho_{t}\left(x_{n}, x_{m}\right)=0$ for each $t \in(0,1]$ and all $n, m \in \mathbb{N}$. For any $t \in(0,1]$, there exists $s=s(t) \in(0, t]$, and we have

$$
\begin{aligned}
\rho_{t}\left(x_{n}, x_{m}\right)= & \rho_{t}\left(f x_{n-1}, f x_{m-1}\right) \\
\leq & (1-\varepsilon) \frac{1}{2}\left(\rho_{t}\left(x_{n}, x_{n+1}\right)+\rho_{t}\left(x_{m}, x_{m+1}\right)\right) \\
& +\Lambda \varepsilon^{\alpha-1} \psi(\varepsilon)\left[1+\left\|x_{n-1}\right\|_{t}+\left\|x_{m-1}\right\|_{t}+\left\|x_{n}\right\|_{t}\right. \\
& \left.+\left\|x_{m}\right\|_{t}\right]^{\alpha} \\
\leq & (1-\varepsilon) \frac{1}{2}\left(\rho_{t}\left(x_{n}, x_{n+1}\right)+\rho_{t}\left(x_{m}, x_{m+1}\right)\right) \\
& +\Lambda \varepsilon^{\alpha-1} \psi(\varepsilon)\left[1+4 M_{t}\right]^{\alpha} .
\end{aligned}
$$

Putting $n \longrightarrow \infty$ and $\varepsilon \longrightarrow 0$ in (36), we get $\rho_{t}\left(x_{n}, x_{m}\right) \longrightarrow 0$.

Therefore, $\left\{x_{n}\right\}$ is a Cauchy sequence. Since $X$ is complete, there exists $z \in X$ such that $x_{n} \longrightarrow z(n \longrightarrow \infty)$.

Step 5. We prove that $z$ is the unique fixed point for mapping $f$.

First, we show that $f z=z$. Using (29), for any $t \in(0,1]$, there exists $s \in(0, t]$, and we have

$$
\begin{aligned}
\rho_{t}(f z, z) \leq & \rho_{s}\left(x_{n+1}, z\right)+\rho_{t}\left(f z, x_{n+1}\right) \\
\leq & (1-\varepsilon) \frac{1}{2}\left(\rho_{t}(z, f z)+\rho_{t}\left(x_{n}, x_{n+1}\right)\right) \\
& +\Lambda \varepsilon^{\alpha} \psi(\varepsilon)\left(1+\|z\|_{t}+\left\|x_{n}\right\|_{t}+\|f z\|_{t}+\left\|x_{n+1}\right\|_{t}\right)^{\alpha} \\
& +\rho_{s}\left(x_{n+1}, z\right) \\
\leq & (1-\varepsilon) \frac{1}{2}\left(\rho_{t}(z, f z)+\rho_{t}\left(x_{n}, x_{n+1}\right)\right)+\Lambda \varepsilon^{\alpha} \psi(\varepsilon) \\
& \left(1+4 M_{t}\right)^{\alpha}+\rho_{s}\left(x_{n+1}, z\right) .
\end{aligned}
$$

By taking limits as $n \longrightarrow \infty$ and $\varepsilon \longrightarrow 0$ in the inequality above, we get $\rho_{t}(f z, z) \leq(1 / 2) \rho_{t}(f z, z)$. Hence, $\rho_{t}(f z, z)=$ 0 which implies $f z=z$.

Finally, we prove the uniqueness of $z$. Assume that $z^{\prime}$ is another fixed point of $f$. For any $t \in(0,1]$, there exists $s \in(0, t]$, and we get

$$
\begin{aligned}
\rho_{t}\left(z, z^{\prime}\right)= & \rho_{t}\left(f z, f z^{\prime}\right) \\
\leq & (1-\varepsilon) \frac{1}{2}\left(\rho_{t}(z, f z)+\rho_{t}\left(z^{\prime}, f z^{\prime}\right)\right)+\Lambda \epsilon^{\alpha} \psi(\varepsilon) \\
& \cdot\left[1+\|z\|_{t}+\left\|z^{\prime}\right\|_{t}+\|f z\|_{t}+\left\|f z^{\prime}\right\|_{t}\right]^{\alpha} \\
\leq & (1-\varepsilon) \frac{1}{2}\left(\rho_{t}(z, z)+\rho_{t}\left(z^{\prime}, z^{\prime}\right)\right)+\Lambda \epsilon^{\alpha} \psi(\varepsilon)\left[1+4 M_{t}\right]^{\alpha} \\
\leq & \Lambda \epsilon^{\alpha} \psi(\varepsilon)\left[1+4 M_{t}\right]^{\alpha} .
\end{aligned}
$$

Passing to the limit as $\varepsilon \longrightarrow 0$ in (38), we have $\rho_{t}\left(z, z^{\prime}\right)=0$. Thus, $z=z^{\prime}$. Therefore, $f$ has a unique fixed point $z \in X$. 
Following the arguments in Remark 1, from Theorem 4, we can obtain the Corollary 2 .

Corollary 2 (see Corollary 5.3, [6]). Let $(X, d, L, R)$ be a complete fuzzy metric space with (R-2) and $f: X \longrightarrow X$ be a mapping. If there exists $\lambda \in[0,(1 / 2))$ such that

$$
\rho_{t}(f x, f y) \leq \lambda\left[\rho_{t}(x, f x)+\rho_{t}(y, f y)\right],
$$

for all $t \in(0,1]$ and $x, y \in X$, then $f$ has a unique fixed point $z \in X$.

Now, we construct two examples to illustrate Theorems 3 and 4 , respectively.

Example 1. Let $X=\left\{\left(1 / 2^{n}\right): n \in \mathbb{N} \cup\{0\}\right\} \cup\{0\}, \quad L(a, b)=$ $\min \{a, b\}$, and $R(a, b)=\max \{a, b\}$. Define the fuzzy metric $d: X \times X \longrightarrow \mathscr{F}^{+}$by

$$
d(x, y)=\overline{0}(q-|x-y|)= \begin{cases}1, & q=|x-y|, \\ 0, & q \neq|x-y| .\end{cases}
$$

Let $T: X \longrightarrow X$ be a map defined by $T x=(1 / 2) x$ for $x \in X$. Then, the following hold:

(a) $(X, d, L, R)$ is a complete fuzzy metric with $(R-2)$.

(b) (6) is satisfied for $x_{0}=1, \Lambda=1, \alpha=1$, and $\psi(\varepsilon)=2 \varepsilon$.

(c) $T$ has the unique fixed point $x=0$.

(d) (29) is not satisfied. So, Theorem 4 cannot be verified by this example.

Proof. First, it is easy to see that $(X, d, L, R)$ is a complete fuzzy metric space, and $R(a, b)=\max \{a, b\}$ satisfies $(R-2)$. Note that $T x=x$ implies that $x=0 \in X$; then, conclusion (c) is true. Next, we prove conclusions (b) and (d), respectively.

(b) Letting $x_{0}=1, \Lambda=1, \alpha=1, \beta=1$, and $\psi(\varepsilon)=2 \varepsilon$, we show that (6) holds for all $\varepsilon \in[0,1], t \in(0,1]$ and $x, y \in X$.

For any $t \in(0,1]$, we can see that $\rho_{t}(x, y)=|x-y|$. Then, we consider the following two cases:

Case 1. If $\varepsilon \leq(1 / 2)$, then $1-\varepsilon \geq(1 / 2)$. For any $x, y \in X$, we have

$$
\begin{aligned}
\rho_{t}(T x, T y)= & \left|\frac{1}{2} x-\frac{1}{2} y\right|=\frac{1}{2}|x-y| \leq(1-\varepsilon) \rho_{t}(x, y) \\
\leq & (1-\varepsilon) \rho_{t}(x, y)+\varepsilon \psi(\varepsilon)\left(1+\|x\|_{t}+\|y\|_{t}\right. \\
& \left.+\|T x\|_{t}+\|T y\|_{t}\right) .
\end{aligned}
$$

Case 2. If $\varepsilon>(1 / 2)$, then $\psi(\varepsilon)>1$. Note that

$$
\rho_{t}(x, y) \leq \rho_{t}\left(x, x_{0}\right)+\rho_{0}\left(x_{0}, y\right)=\|x\|_{t}+\|y\|_{t} .
$$

Then, for any $x, y \in X$, we have

$$
\begin{aligned}
\rho_{t}(T x, T y)= & \left|\frac{1}{2} x-\frac{1}{2} y\right| \leq|x-y|=(1-\varepsilon) \rho_{t}(x, y)+\varepsilon \rho_{t}(x, y) \\
\leq & (1-\varepsilon) \rho_{t}(x, y)+\varepsilon \psi(\varepsilon)\left(\|x\|_{t}+\|y\|_{t}\right) \\
\leq & (1-\varepsilon) \rho_{t}(x, y)+\varepsilon \psi(\varepsilon)\left(1+\|x\|_{t}+\|y\|_{t}\right. \\
& \left.+\|T x\|_{t}+\|T y\|_{t}\right) .
\end{aligned}
$$

From Cases 1 and 2, we see that (6) holds for all $\varepsilon \in[0,1]$. In fact, $x=0$ is the unique fixed point for map $T$. So, Theorem 3 is verified.

(d) We show that (29) is not satisfied.

Letting $\varepsilon=0$, by (29), we deduce that

$$
\rho_{t}(T x, T y) \leq \frac{\rho_{t}(x, T x)+\rho_{t}(y, T y)}{2} .
$$

However, let $x_{0}=0$ and $y_{0}=1$. For any $t \in(0,1]$, we have

$$
\rho_{t}\left(T x_{0}, T y_{0}\right)=\left|0-\frac{1}{2}\right|>\frac{1}{2} \cdot \frac{1}{2}=\frac{\rho_{t}\left(x_{0}, T x_{0}\right)+\rho_{t}\left(y_{0}, T y_{0}\right)}{2} \text {. }
$$

So, this example cannot verify Theorem 4 , and we prove conclusion (d).

Example 2. Let $X=\{6,-5,4,-3,2,-1,0\}, \quad L(a, b)=\min$ $\{a, b\}$, and $R(a, b)=\max \{a, b\}$. Let $d: X \times X \longrightarrow \mathscr{F}^{+}$be a fuzzy metric such that

$$
d(x, y)(q)= \begin{cases}0, & q<0 \\ 1, & q=0 \\ \frac{|x-y|}{|x-y|+q}, & q>0 .\end{cases}
$$

Let $T: X \longrightarrow X$ be a map such that

$$
T x= \begin{cases}-|x|+1, & x>0, \\ |x|-1, & x<0, \\ 0, & x=0,\end{cases}
$$

for $x \in X$. Then, the following hold:

(a) $(X, d, L, R)$ is a complete fuzzy metric with $(R-2)$.

(b) (29) is satisfied for $x_{0}=1, \Lambda=1, \alpha=1$, and $\psi(\varepsilon)=6 \varepsilon$.

(c) $T$ has the unique fixed point $x=0$.

(d) (6) is not satisfied. So, this example cannot verify Theorem 3.

Proof. Clearly, we can see that $x=0$ is the unique fixed point for map $T$, and conclusion (c) is true. Now, we prove conclusions (a), (b), and (d), respectively. 
(a) We show that $(X, d, L, R)$ is a complete fuzzy metric space with $(R-2)$.

Clearly, for any $x, y \in X, d(x, y)(q)$ is a fuzzy number, and (D1) and (D2) in Definition 2 are satisfied. Note that $R(a, b)=\max \{a, b\}$ satisfies (R-2). So, it is sufficient to prove that (D3) holds.

For any $x, y, z \in X$, it is obvious that $\lambda_{1}(x, z)=$ $\lambda_{1}(z, y)=\lambda_{1}(x, y)=0$. To see (D3L), let us consider the following two cases:

(i) If $p<0$ or $q<0$, then $d(x, z)(p)=0$ or $d(z, y)(q)=0$, leading to that

$$
\min (d(x, z)(p), d(z, y)(q))=0 .
$$

So, (D3L) is satisfied.

(ii) If $p=0$ and $q=0$, then $p+q=0$. So, $d(x, z)(p)=d(z, y)(q)=d(x, y)(p+q)=1$, leading to that

$$
d(x, y)(p+q)=\min \{d(x, z)(p), d(z, y)(q)\} .
$$

Thus, (D3L) holds. To see (D3R), we consider the following two cases:

Case a1. Suppose that there are at least two points in $\{x, y, z\}$ which are equal. For any $p \geq 0$ and $q \geq 0$,

(i) If $z=x$ or $z=y$, without loss of generality, let $z=x$, then $d(z, y)=d(x, y)$. Since $d(x, y)(q)$ is decreasing as $q \geq 0$, we have

$$
\begin{aligned}
\max & \{d(x, z)(p), d(z, y)(q)\} \geq d(z, y)(q) \\
& =d(x, y)(q) \geq d(x, y)(p+q) .
\end{aligned}
$$

(ii) If $x=y$, then we have

$$
\begin{aligned}
p+q & =0 \Rightarrow p=0, q=0 \Rightarrow d(x, y)(p+q) \\
& =1=\max \{d(x, z)(p), d(z, y)(q)\} . \\
p+q>0 & \Rightarrow d(x, y)(p+q)=0 \\
& \leq \max \{d(x, z)(p), d(z, y)(q)\} .
\end{aligned}
$$

Case a2. Suppose that $x \neq y, x \neq z$, and $y \neq z$. For any $p \geq 0$ and $q \geq 0$, denote $s=d(x, z)(p)$ and $t=d(z, y)(q)$. Without loss of generality, let $0<s \leq t$. Then, we have

$$
\begin{aligned}
& p=|x-z| \frac{1-s}{s}, \\
& q=|z-y| \frac{1-t}{t} .
\end{aligned}
$$

Since $d(x, y)(q)$ is decreasing as $q \geq 0$ and $(1-t / t)$ is decreasing, we deduce that

$$
\begin{aligned}
\max \{d(x, z)(p), d(z, y)(q)\} & =t=d(x, y)\left(|x-y| \frac{1-t}{t}\right) \\
& \geq d(x, y)\left[(|x-z|+|z-y|) \frac{1-t}{t}\right] \\
& \geq d(x, y)\left[|x-z| \frac{1-s}{s}+|z-y| \frac{1-t}{t}\right] \\
& =d(x, y)(p+q) .
\end{aligned}
$$

From Cases al and a2, we conclude that (D3R) holds for all $x, y, z \in X$.

Note that $X$ is a finite set. Then, $(X, d, L, R)$ is complete, and the proof of conclusion (a) is completed.

(b) We prove (29) for $x_{0}=1, \Lambda=1, \alpha=1, \beta=1$, and $\psi(\varepsilon)=6 \varepsilon$.

For any $t \in(0,1]$, we can see that $\rho_{t}(x, y)=$ $|x-y|(1-t / t)$. Then, we consider the following two cases:

Case b1. If $\varepsilon \leq(1 / 12)$, it is easy to see that, for any $x \neq 0$, we have $|T x|=|x|-1$ and $x \cdot T x \leq 0$. So, we can obtain that $|x-T x|=|x|+|T x|$ and

$$
\begin{aligned}
|T x-T y| \leq|T x|+|T y| & =\frac{|T x|+|x|-1}{2}+\frac{|T y|+|y|-1}{2} \\
& =\frac{|x-T x|+|y-T y|}{2}-1
\end{aligned}
$$

for all $x, y \neq 0$. If $y=0$, then we have

$$
\begin{aligned}
|T x-T y| & =|T x|=\frac{|T x|+|x|-1}{2} \\
& =\frac{|x-T x|+|y-T y|}{2}-\frac{1}{2} .
\end{aligned}
$$

From the above two equalities, by calculation, we obtain that, for any $x, y \in X$,

$$
\begin{aligned}
\rho_{t}(T x, T y) & =|T x-T y| \frac{1-t}{t} \leq \frac{10}{11} \cdot \frac{|x-T x|(1-t / t)+|y-T y|(1-t / t)}{2} \\
& \leq(1-\varepsilon) \frac{\rho_{t}(x, T x)+\rho_{t}(y, T y)}{2} \\
& \leq(1-\varepsilon) \frac{\rho_{t}(x, T x)+\rho_{t}(y, T y)}{2}+\varepsilon \psi(\varepsilon)\left(1+\|x\|_{t}+\|y\|_{t}+\|T x\|_{t}+\|T y\|_{t}\right) .
\end{aligned}
$$


Case b2. If $\varepsilon>(1 / 12)$, then we have $\psi(\varepsilon)>(1 / 2)$. Note Then, for any $x, y \in X$, we have that

$$
\begin{aligned}
\rho_{t}(x, T x)+\rho_{t}(y, T y) \leq & \rho_{t}\left(x, x_{0}\right)+\rho_{t}\left(x_{0}, T x\right) \\
& +\rho_{t}\left(y, x_{0}\right)+\rho_{t}\left(x_{0}, T y\right) \\
= & \|x\|_{t}+\|y\|_{t}+\|T x\|_{t}+\|T y\|_{t} .
\end{aligned}
$$

$$
\begin{aligned}
\rho_{t}(T x, T y) & =|T x-T y| \frac{1-t}{t} \leq \frac{|x-T x| 1-t / t+|y-T y| 1-t / t}{2} \\
& =(1-\varepsilon) \frac{\rho_{t}(x, T x)+\rho_{t}(y, T y)}{2}+\varepsilon \frac{\rho_{t}(x, T x)+\rho_{t}(y, T y)}{2} \\
& \leq(1-\varepsilon) \frac{\rho_{t}(x, T x)+\rho_{t}(y, T y)}{2}+\varepsilon \frac{1}{2}\left(\|x\|_{t}+\|y\|_{t}+\|T x\|_{t}+\|T y\|_{t}\right) \\
& \leq(1-\varepsilon) \frac{\rho_{t}(x, T x)+\rho_{t}(y, T y)}{2}+\varepsilon \psi(\varepsilon)\left(1+\|x\|_{t}+\|y\|_{t}+\|T x\|_{t}+\|T y\|_{t}\right) .
\end{aligned}
$$

From Cases b1 and b2, we see that (29) holds for all $\varepsilon \in[0,1]$. In fact, $x=0$ is the unique fixed point for map $T$. So, Theorem 4 is verified.

(d) We show that (6) is not satisfied for map $T$ in $(X, d, L, R)$.

Finally, we show that (6) is not satisfied for map $T$ in $(X, d, L, R)$. If $\rho_{t}(T x, T y)=\rho_{t}(x, y)$ for some $x, y \in X$ and some $t \in(0,1]$, then by $(6)$, we deduce that, for any $\varepsilon>0$,

$$
\rho_{t}(x, y) \leq \Lambda \varepsilon^{\alpha} \psi(\varepsilon)\left[1+\|x\|_{t}+\|y\|_{t}+\|f x\|_{t}+\|f y\|_{t}\right]^{\beta} .
$$

Letting $\varepsilon \rightarrow 0$, we have $\psi(\varepsilon) \rightarrow 0$, which implies that $x=y$. However, for $x_{0}=6$ and $y_{0}=4$, we have $T x_{0}=-5$ and $T y_{0}=-3$ and

$$
\rho_{t}\left(T x_{0}, T y_{0}\right)=2 \cdot \frac{1-t}{t}=\rho_{t}\left(x_{0}, y_{0}\right)
$$

for all $t \in(0,1]$, which is a contradiction.

Remark 2. From Examples 1 and 2, we can see that Theorems 3 and 4 are independent to each other.

Recently, Jamshaid et al. [19] investigated the fuzzy fixed points of fuzzy mappings via F-contractions. On the basis of their work, a natural question for multivalued mappings can be raised as follows:

Question 1. Is the multivalued case of Theorem 3 true?

\section{Data Availability}

No data were used to support this study.

\section{Conflicts of Interest}

The authors declare that they have no conflicts of interest.

\section{Authors' Contributions}

All authors contributed equally and significantly in writing this article. All authors read and approved the final manuscript.

\section{Acknowledgments}

This research was supported by the National Natural Science Foundation of China (11561049).

\section{References}

[1] O. Kaleva and S. Seikkala, "On fuzzy metric spaces," Fuzzy Sets and Systems, vol. 12, no. 3, pp. 215-229, 1984.

[2] O. Hadzic and E. Pap, "A fixed point theorem for multivalued mappings in probaailistic metric spaces and an application in fuzzy metric spaces," Fuzzy Sets and Systems, vol. 127, no. 3, pp. 333-444, 2002.

[3] N. Lu, F. He, and J. Fan, "Fixed point theorems of cycliccontractive mapping in fuzzy metric spaces," Mathematics in Practice and Theory, vol. 22, pp. 237-248, 2018.

[4] M. A. Ahmed, "Fixed point theorems in fuzzy metric spaces," Journal of the Egyptian Mathematical Society, vol. 22, no. 1, pp. 59-62, 2014.

[5] G. Yun, S. Hwang, and J. Chang, "Fuzzy lipschitz maps and fixed point theorems in fuzzy metric spaces," Fuzzy Sets and Systems, vol. 161, no. 8, pp. 1117-1130, 2010.

[6] J.-Z. Xiao, X.-H. Zhu, and X. Jin, "Fixed point theorems for nonlinear contractions in Kaleva-Seikkala's type fuzzy metric spaces," Fuzzy Sets and Systems, vol. 200, pp. 65-83, 2012.

[7] S. Phiangsungnoen, P. Thounthong, and P. Kumam, "Fixed point results in fuzzy metric spaces via $\alpha$ and $\beta \kappa$-admissible mappings with application to integral types," Journal of Intelligent \& Fuzzy Systems, vol. 34, no. 1, pp. 467-475, 2018.

[8] G.-J. Yun, J. Chang, and S. Hwang, "Fuzzy isometries and non-existence of fuzzy contractive maps on fuzzy metric spaces," International Journal of Fuzzy Systems, vol. 13, no. 3, pp. 206-217, 2011. 
[9] V. Gregori, J.-J. Miñana, and D. Miravet, "Contractive sequences in fuzzy metric spaces," Fuzzy Sets and Systems, vol. 379, pp. 125-133, 2020.

[10] S. S. Chauhan, M. Imdad, G. Kaur, and A. Sharma, "Some fixed point theorems for $S_{F}$-contraction in complete fuzzy metric spaces," Afrika Matematika, vol. 30, no. 3-4, pp. 651-662, 2019.

[11] J. Xiao and X. Zhu, "On linearly topological structure and property of fuzzy normed linear space," Fuzzy Sets and Systems, vol. 125, no. 2, pp. 153-161, 2002.

[12] J.-z. Xiao and X.-h. Zhu, "Topological degree theory and fixed point theorems in fuzzy normed space," Fuzzy Sets and Systems, vol. 147, no. 3, pp. 437-452, 2004.

[13] V. Pata, "A fixed point theorem in metric spaces," Journal of Fixed Point Theory and Applications, vol. 10, no. 2, pp. 299305, 2011.

[14] M. Chakraborty and S. K. Samanta, "A fixed point theorem for Kannan-type maps in metric spaces,” 2012, https://arxiv.org/ abs/1211, 7331v2.

[15] Z. Kadelburg and S. Radenović, "Fixed point theorems under Pata-type conditions in metric spaces," Journal of the Egyptian Mathematical Society, vol. 24, no. 1, pp. 77-82, 2016.

[16] Z. Kadelburg and S. Radenovic, "Fixed point and tripled fixed point theorems under pata-type conditions in ordered metric spaces," International Journal of Analysis and Applications, vol. 6, pp. 113-122, 2014.

[17] S. Balasubramanian, "A pata-type fixed point theorem," Mathematical Sciences, vol. 8, no. 3, pp. 65-69, 2014.

[18] G. K. Jacob, M. S. Khan, C. Park, and S. Jun, "On generalized pata type contractions," Mathematics, vol. 6, no. 2, p. 25, 2018.

[19] J. Ahmad, H. Aydi, and N. Mlaiki, "Fuzzy fixed points of fuzzy mappings via F-contractions and an application," Journal of Intelligent \& Fuzzy Systems, vol. 37, no. 4, pp. 5487-5493, 2019. 